\title{
Relação do paciente com o serviço em Unidades Básicas de Saúde sob a óptica dos médicos e dos pacientes
}

\author{
Doctors' and patients' views of patients' relationship with primary care practice services
Relación del paciente con los servicios en las Unidades Básicas de Salud de acuerdo con la visión de los médicos y de los pacientes

Fernando de Almeida Machado. Universidade Federal do Tocantins (UFT). Palmas, TO, Brasil. fam@uft.edu.br (Autor correspondente)

Ranielly Ribeiro Venturini. Universidade Federal do Tocantins (UFT). Palmas, TO, Brasil. ranyventurini@ hotmail.com Ariadnne Lino Alcantara Manzan. Universidade Federal do Tocantins (UFT). Palmas, TO, Brasil. ariadnnemanzan@ hotmail.com Grazielle Rodrigues Silva. Universidade Federal do Tocantins (UFT). Palmas, TO, Brasil. grazielle.rs@gmail.com

\section{Resumo}

Objetivo: discutir aspectos relacionados à relação do paciente com o serviço em Unidades Básicas de Saúde sob a óptica dos médicos e dos pacientes, avaliando as suas expectativas e relacionando-as à subjetividade que permeia a prática médica. Métodos: estudo qualitativo desenvolvido por meio de entrevistas estruturadas e aplicadas a médicos e pacientes de oito Unidades Básicas de Saúde do município de Palmas (TO). Resultados: muitos pacientes não têm suas expectativas satisfeitas e grande parte dessa insatisfação tem por origem problemas na relação médico-paciente. De modo geral, o médico valoriza a evolução clínica e o tratamento instituído e preocupa-se com aspectos operacionais do seu trabalho, ao passo que o paciente tem expectativas ligadas a aspectos mais subjetivos, inseridos dentro de variáveis psicossociais, necessidades muitas vezes negligenciadas pelo médico. Conclusão: o trabalho aponta, diretamente, para a necessidade de estabelecer condições que favoreçam o surgimento de uma boa relação profissional-paciente, sem a qual não há recuperação plena da saúde e, indiretamente, para a importância dessa discussão em relação à formação acadêmica, deixando evidente a necessidade de se trabalhar competências e habilidades dentro desse aspecto.

\section{Abstract}

Objective: the objective of this study was to discuss patients' relationship with health services from the viewpoint of doctors and patients, considering the expectations of both parties and the subjectivity that permeates medical practice. Methods: data for this qualitative study were collected through structured interviews with doctors and patients from eight primary health care units in the city of Palmas, Tocantins. Results: the expectations of many patients were not satisfied due to problems in the relationships with their doctors. Generally, doctors overestimated clinical evolution and instituted treatment that was focused on the operational aspects of their work, whereas patients had more subjective expectations relating to psychosocial variables that were disregarded by the doctors. Conclusion: this study indicates that there is a need to establish conditions that promote good relationships between doctors and patients, and that this is essential to the patients' full health recovery. The results also suggest that it is very important for medical schools to provide future health care professionals with skills to better deal with the human aspects of their practice.
Como citar: Machado FA, Venturini RR, Manzan ALA, Silva GR. Relação do paciente com o serviço em Unidades Básicas de Saúde sob a óptica dos médicos e dos pacientes. Rev Bras Med Fam Comunidade. 2015;10(37):0-00. http://dx.doi.org/10.5712/rbmfc10(37)773
Palavras-chave:

Relações Médico-Paciente

Satisfação do Paciente

Atenção Primária à Saúde

Serviços de Saúde

Centros de Saúde

\section{Keywords:}

Physician-Patient Relationships Patient Satisfaction

Primary Care Practices Health Services Health Centers

Fonte de financiamento: declaram não haver. Parecer CEP: 023/2009 (UFT), aprovado em 08/07/2009.

Conflito de interesses: declaram não haver.

Procedência e revisão por pares: revisado por pares. Recebido em: 09/06/2013. Aprovado em: 27/04/2015. 


\section{Resumen}

Objetivo: discutir los aspectos relacionados a la relación de los pacientes con el servicio de salud bajo la óptica de los médicos y de los pacientes, evaluando sus expectativas, teniendo en consideración la subjetividad que intervienen la práctica médica. Métodos: estudio cualitativo desarrollado por medio de entrevistas estructuradas y aplicadas a médicos y a pacientes de ocho Unidades Básicas de Salud de la municipalidad de Palmas (TO). Resultados: muchos pacientes no tienen sus expectativas satisfechas y gran parte de esta insatisfacción tiene por origen problemas en la relación médico-paciente. De una manera general, el médico valoriza la evolución clínica y el tratamiento instituido y se preocupa con los aspectos operacionales de su trabajo al paso que el paciente tiene expectativas que están relacionadas a aspectos más subjetivos, insertados dentro de variables psicosociales, necesidades muchas veces descuidadas por el médico. Conclusión: el trabajo apunta, directamente, para la necesidad de establecer condiciones que favorezcan al surgimiento de una buena relación médico-paciente, sin la cual no hay recuperación plena de la salud e, indirectamente, para la importancia de esta discusión en relación a la formación académica, dejando evidente la necesidad de trabajarse las competencias y habilidades adentro de este aspecto.

\section{Palabras clave:}

Relaciones Médico-Paciente Satisfacción de los Pacientes Servicios de Salud Centros de Salud Unidades Básicas de Salud.

\section{Introdução}

Os conceitos de saúde e doença sofrem modificações ao longo da história, de acordo com a evolução do pensamento e da cultura dos povos, da evolução social e do impulso dado pelo conhecimento e controle da tecnologia. De uma maneira geral, principalmente a partir do século $X X$, as faculdades de medicina passaram a apresentar um ensino baseado no modelo biomédico, cuja formação estimula a compreensão da doença em si e valoriza uma abordagem terapêutica padronizada. Não é exagero afirmar que, como consequência desse pensamento, o conhecimento das doenças passou a ser mais valorizado do que a assistência ao doente. Sem desconsiderar os excelentes avanços científicos e tecnológicos conseguidos pelo modelo biomédico, fundamentais para a melhoria dos indicadores de saúde e para a melhoria da qualidade de vida, é preciso atenção para não se negligenciar outros aspectos interdependentes da saúde humana. ${ }^{1,2}$

Esse modelo tradicional e consolidado tem por base uma atitude centrada no médico ou na doença, não conferindo uma relevância necessária ao indivíduo dentro de uma análise holística. O doente deixa de ser o complexo, atribuindo-se a complexidade à doença em si. O conhecimento complexo, nos dizeres de Minayo, ${ }^{3}$ é a essência para se conhecer os seres vivos, que são diferentes sistemas entrelaçados em suas circunstâncias.

Provavelmente, a postura médica tecnicista ainda predominante explique um aparente paradoxo: embora se tenha conseguido tanto conhecimento nas diversas áreas das ciências da saúde em tão pouco tempo e atualmente se viva uma época de extraordinário desenvolvimento tecnológico, existe um descontentamento social em relação ao profissional médico e à prática da medicina. ${ }^{4}$

Balint ${ }^{5}$ considera a importância da compreensão plena do homem em suas diferentes dimensões e o uso adequado dessa compreensão para a promoção da saúde. Evidentemente, para que isto seja alcançado, é necessário que se estabeleça uma relação médico-paciente adequada, satisfazendo aos anseios de ambas as partes.

A relação entre o profissional médico e o paciente vem se transformando com o tempo. A forma como é constituído esse complexo processo de interação entre o médico e a pessoa influencia no resultado do trabalho médico e ocorre na dependência da habilidade e competência do profissional em adequar-se às queixas, demandas, expectativas, crenças e emoções de cada paciente. ${ }^{6}$

Apesar da importância da abordagem holística do paciente no sentido de aperfeiçoar a relação entre ele e o médico, as grades curriculares da maioria das escolas médicas não contemplam a busca de 
competências técnicas que aprimorem a formação humanística do aluno. Pesquisas que envolvem a relação médico-paciente durante a formação médica são escassas. ${ }^{7}$ Possivelmente, uma mudança pedagógica na qual o ensino esteja centrado no aluno seja uma estratégia para educar o futuro profissional a valorizar a abordagem centrada na pessoa. ${ }^{8}$

A literatura especializada nacional registra relativamente poucos estudos sobre o tema da relação médico-paciente, e muitas dessas publicações nacionais são revisões ou ensaios sobre teorias pré-definidas. ${ }^{9}$ Em geral, existe um predomínio de estudos quantitativos a respeito desse tema, embora mais recentemente perceba-se uma tendência em publicações sobre o assunto dentro de uma perspectiva qualitativa ou mesmo combinando métodos quantitativos e qualitativos. ${ }^{10,11}$

O Estado do Tocantins foi criado pela Constituição de 1988 e tem atraído médicos de diferentes regiões do país, assim como um grande contingente de pessoas procedentes de diversas regiões da nação, com cultura, costumes e hábitos diversos. Tais aspectos justificam estudar a relação médico-paciente na cidade de Palmas, capital do Tocatins. Com esta preocupação, o presente estudo objetiva avaliar a relação médico-paciente em Unidades Básicas de Saúde (UBS) do município de Palmas sob a óptica do médico e do usuário e levantar aspectos que permitam uma avaliação tanto dos sucessos quanto das dificuldades que permeiam essa relação.

\section{Métodos}

Esta é uma pesquisa descritivo-exploratória que tem por objetivo avaliar a relação médico-paciente por meio de entrevistas baseadas em um questionário estruturado, com perguntas abertas, aplicadas a médicos e pacientes das Unidades Básicas de Saúde (UBS) das regiões Norte, Central e Sul do município de Palmas (TO), conforme a divisão estabelecida pela Secretaria Municipal de Saúde.

Para a seleção da amostra, foi realizado um levantamento total de UBS do município. Das 35 existentes, foram escolhidas oito, distribuídas nas três regiões que compõem a cidade de Palmas (Norte, Central e Sul), dando preferência às unidades com maior abrangência de quadras atendidas ou com maior número de médicos efetivados. Dessas UBS, duas eram na região norte, três unidades na região central e três unidades na região sul.

Foram entrevistados 48 pacientes com idade acima de 18 anos, isentos de alguma dificuldade de compreensão, expressão verbal ou alteração do estado de consciência, que haviam acabado de passar por uma consulta com o médico da respectiva Unidade. Os 11 médicos entrevistados eram profissionais que estavam efetivados nas UBS, trabalhando regularmente nesses locais há pelo menos um mês da data da entrevista. O número de entrevistados não foi estabelecido a princípio, sendo determinado por critério de saturação de dados à medida que a pesquisa prosseguia, tomando-se como critério o aparecimento de um padrão repetitivo de respostas.

Foram elaborados dois tipos de questionários, um para os usuários e outro para os médicos, ambos com 11 questões. Os questionários seguiram uma ordem de perguntas que envolviam, inicialmente, uma abordagem social, passando para informações sobre a UBS, e sequencialmente para a análise sobre a consulta, sobre o relacionamento médico-paciente naquele serviço e, por fim, sugestões para possíveis mudanças no atendimento prestado naquele local. As entrevistas foram gravadas, transcritas e, posteriormente, interpretadas e agrupadas por ideias. 
Questionário aplicado aos usuários das Unidades Básicas de Saúde de Palmas (TO)

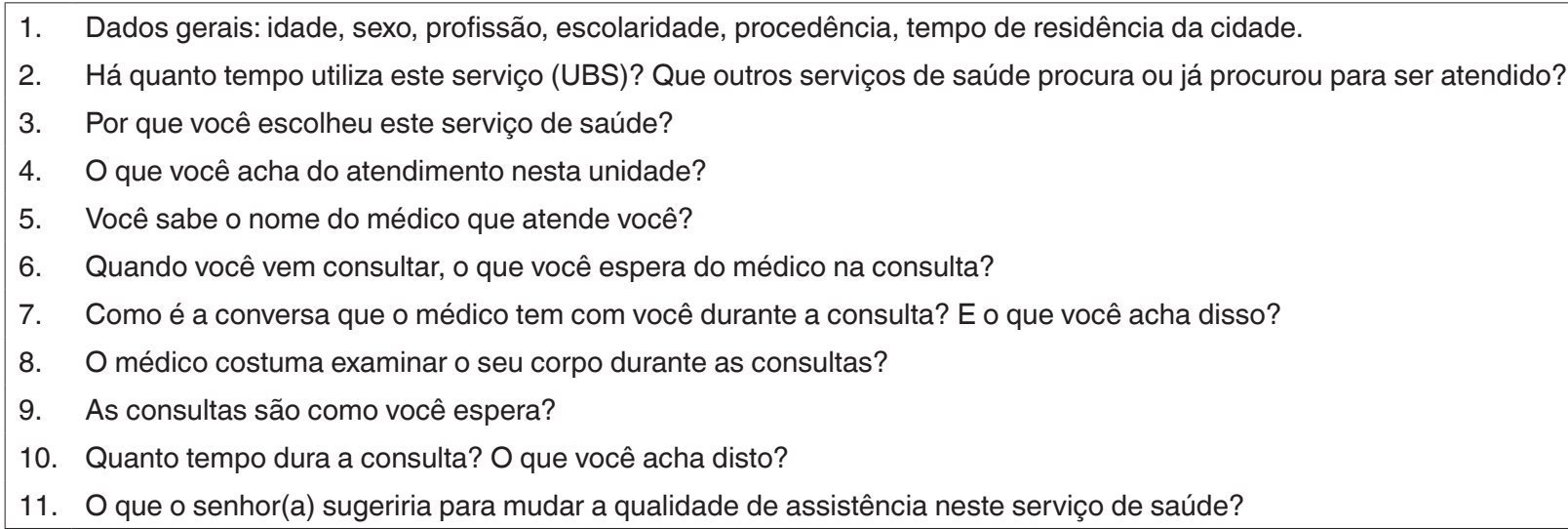

O projeto foi aprovado pelo Comitê de Ética e Pesquisa da Universidade Federal do Tocantins, processo no 023/2009 e as entrevistas foram realizadas com o consentimento documentado pelos entrevistados por meio da assinatura do Termo de Consentimento Livre e Esclarecido.

\section{Resultados e Discussão}

A relação médico-paciente é largamente considerada como a essência na efetividade dos cuidados de saúde. Esta relação envolve uma confidencialidade entre o profissional e um indivíduo vulnerável, caracterizando uma das formas mais importantes das relações humanas, e invoca sentimentos nas suas mais diferentes formas. Por isto, vários trabalhos publicados nos últimos anos sobre a temática enfocam justamente o lado humanístico da relação médico-paciente e a importância na qualidade desta relação no estabelecimento do vínculo do paciente com os cuidados à saúde.,2,9

A grande maioria dos usuários entrevistados nas UBS referiu usar exclusivamente aquele serviço quando buscam atenção à sua saúde. Poucos indivíduos afirmaram já ter consultado dentro de um atendimento em serviço privado. Quando questionados abertamente sobre o motivo de usarem aquele serviço, as respostas basicamente se concentraram em dois argumentos: por falta de outra alternativa, respondida por 17 entrevistados, e pela facilidade de acesso, respondida por 16. Apenas cinco usuários afirmaram consultar naquele serviço por gostarem (do serviço e/ou do médico) e quatro por estarem 
inseridos num dos programas de saúde. Interessa destacar que apenas dois entrevistados argumentaram consultar naquela UBS por considerarem um direito utilizar o serviço oferecido ("acho que se tem um posto de saúde e médico aqui nesta quadra, a gente tem o direito de usar, não é?").

Os entrevistados avaliaram relativamente bem o atendimento nas UBS que habitualmente usavam, apesar dos motivos alegados pela maioria dos pacientes não fazerem referência à qualidade ou satisfação em usar aquele serviço. Para 25 usuários, o atendimento na unidade é bom e apenas 3 entrevistados o classificaram como ruim. Os demais 20 usuários consideraram o atendimento como regular. Os motivos da insatisfação foram relatados espontaneamente por alguns entrevistados e foram diversificados, mas incluíram problemas na relação com os profissionais envolvidos (funcionários e médicos) e queixas a respeito da estrutura física das UBS ("é difícil, a gente fica esperando muito tempo pra ser atendido e ninguém liga pra gente") (... "parece que o pessoal que trabalha aqui está sempre de mau humor"). Como a assistência à saúde é um processo complexo, constituída por muitos componentes, é difícil também a avaliação de sua qualidade.

Estudos mostram que a satisfação dos usuários com os serviços de saúde é componente importante na avaliação desses serviços, embora muitos desses estudos tenham suas metodologias discutidas. ${ }^{12} \mathrm{O}$ modo como as pessoas percebem o serviço de saúde e suas necessidades nem sempre são considerados pelos gestores dos serviços de saúde: muitas vezes, as atividades e os programas desenvolvidos nesses serviços respeitam mais as necessidades técnico-políticas. ${ }^{13} \mathrm{E}$ um dos determinantes mais importantes na avaliação da qualidade dos serviços de saúde e na vinculação do paciente ao serviço é justamente a relação médico-paciente. ${ }^{9}$ Em estudo que objetivou avaliar fatores associados à frequência da utilização do serviço de puericultura, autores nacionais constataram que, de um total de 198 mães ou responsáveis, 21,7\% não levaram as crianças às consultas por problemas com o serviço, dentre os quais a insatisfação com o atendimento dos profissionais. ${ }^{14} \mathrm{Em}$ outro estudo nacional, observa-se uma maior vinculação dos usuários ao serviço de saúde com a qualidade da atenção, avaliada por diferentes meios relacionados à eficácia da comunicação, como conhecer o motivo da consulta e o nome do médico que faz o atendimento. ${ }^{15} \mathrm{Em}$ relação a este último aspecto, é importante destacar que, na presente casuística, $55,5 \%$ dos entrevistados não souberam referir o nome do médico que os havia atendido.

Questionados sobre suas expectativas a respeito do médico na consulta, as respostas mais apresentadas pelos pacientes referiram-se à atenção e orientação adequadas, a um bom atendimento e paciência, satisfação de suas dúvidas, prescrição de medicamentos e solicitação de exames ("a gente espera que ele tenha paciência para ouvir as queixas da gente para poder ajudar"; "eu espero que ele me dê atenção e peça os exames para saber o que eu tenho"). Apenas dois entrevistados disseram que suas expectativas residiam na resolução de seus problemas, enquanto a grande maioria dos entrevistados mencionou a atenção do médico como uma de suas principais expectativas. Essa manifestação, que atribui grande importância à atenção médica na qualidade da consulta, mostra a necessidade que o paciente tem em ser ouvido quanto aos seus anseios.

Alguns entrevistados referiram a prescrição de medicamentos dentro da expectativa que têm dos médicos na consulta. É possível entender que alguns entrevistados tenham dado importância aos medicamentos prescritos como um reflexo da cultura histórica do modelo médico-assistencialista, que 
valoriza as medidas de caráter curativo e sustenta a atitude "medicalizante" como solução dos problemas de saúde. A relação estabelecida entre a indústria farmacêutica com os médicos, muitos deles completamente enquadrados às exigências mercadológicas, reforça esse modelo. ${ }^{16}$

Quando questionados se as consultas eram como se esperava, houve divisão exata entre os usuários que responderam afirmativamente e aqueles que disseram que não ou nem sempre, excluindo-se seis pacientes que não souberam responder. Embora as expectativas dos pacientes sobre o médico recaiam mais em aspectos subjetivos do comportamento profissional, como na atenção médica dispensada, praticamente metade dos usuários respondentes refere frustrações com a consulta.

Esta insatisfação não é um problema apenas local. Muitos estudos revelam a insatisfação de pacientes quanto à atenção recebida durante a consulta médica, evidenciada principalmente na baixa disposição de tempo e na falta de paciência do médico em escutar aquilo que o paciente tem a dizer, o que gera frustração na expectativa de estabelecer uma comunicação satisfatória com o profissional. ${ }^{17}$ Entre os usuários entrevistados, muitos atribuem aos profissionais médicos e à estruturação de suas consultas a sensação de indiferença e de pouco vínculo com os problemas que os afligem ("... às vezes, a gente tá sentindo alguma coisa e às vezes a gente quer falar e eles não escutam o que a gente tem para falar"). Pode-se dizer que a atitude médica dentro do tradicional modelo biomédico, na visão dos usuários, reforça a postura reducionista, que considera quase exclusivamente a queixa biológica, desconsiderando outros aspectos subjetivos da saúde humana. Em muitas respostas dadas pelos usuários, existe um discurso que remete à humanização do atendimento, tanto enfatizando a sua falta como reconhecendo sua presença.

É frequente os médicos elegerem um problema do paciente para explorar, dificultando a oportunidade de expressar outras queixas e, mesmo assim, nem sempre o paciente consegue completar o relato do problema que o médico julgou ser o principal. Em um estudo desenvolvido por Marvel et al. ${ }^{18}$ foi determinado que o tempo médio utilizado pelos pacientes para relatarem suas queixas antes que os médicos fizessem o primeiro redirecionamento foi de apenas 23 segundos. Esta interrupção precoce não se explica por uma possível invasão no tempo caso o paciente fosse liberado para falar o quanto quisesse, comprometendo a agenda e o andamento do trabalho médico, mas sustenta-se pela própria formação profissional ainda enraizada numa abordagem quase exclusivamente biológica. ${ }^{19,20}$

Questionados sobre o que acham da conversa que o médico tem com eles durante a consulta, a maioria (23) estava satisfeita, sendo que alguns (11) estavam insatisfeitos, poucos (2) responderam que a conversa era regular e outros 12 não souberam qualificar, assumindo a dificuldade de qualificá-la ou dando respostas evasivas, sem que pudessem ser classificadas pelos autores. Por outro lado, indagados se o médico costumava examinar o seu corpo durante a consulta, 20 disseram que não, 14 disseram que sim e 10 disseram que o exame físico era feito algumas vezes. Muitos pacientes que disseram ter sua expectativa atendida na consulta afirmaram que o exame físico ou não era feito ou era feito de maneira superficial.

$\mathrm{Na}$ avaliação da percepção do usuário sobre o tempo de consulta, 16 julgaram muito curto ou insuficiente, 20 julgaram-no suficiente e 12 não souberam avaliar. Os argumentos usados pelos pacientes variaram muito. Alguns consideraram a elevada demanda da UBS para justificar que o médico não pode demorar muito com cada um dos pacientes, embora muitos deles considerem que o profissional médico não cumpra exatamente sua carga horária de trabalho no local e que não seja pontual. Dentre os que 
avaliaram o tempo de consulta suficiente, alguns responderam espontaneamente que o tempo da consulta é curto, mas imposto pela estrutura do serviço, como a demanda na sala de espera ("Ela faz de 3 a 4 minutos. Bom, esse tempo pode ser curto, mas como ela é uma clínica geral, ela só vai te ouvir. É ótimo esse tempo. Tem muita gente para ela atender"). É curioso que, mesmo dentre os que julgam o tempo da consulta suficiente, em outro momento da entrevista fazem críticas contundentes em aspectos que têm como base exatamente a fragilidade da consulta oferecida. Cabe considerar a subjetividade das percepções relacionadas ao vínculo entre o paciente e o profissional, o que mostra que, nos dizeres de Albuquerque e Bosi, ${ }^{21}$ "no plano das experiências com a consulta médica não existe uma homogeneidade".

Em uma tese de doutorado, Arroyo 22 analisou como o tempo de atendimento influencia na avaliação da qualidade dos serviços de saúde pelos usuários. A autora fez seu levantamento em pacientes usuários de três Unidades Básicas de Saúde, quatro hospitais particulares e um hospital público. As instituições de saúde que tiveram pior avaliação de qualidade também apresentaram os menores tempos de consulta médica. Quando as consultas são rápidas e mal estruturadas, os pacientes percebem que não receberam a atenção necessária do médico, mesmo se o médico tem um bom relacionamento com o seu paciente ( “... a minha acho que não chegou nem nos três minutos. Acho isso errado. Não é pra demorar, pra deitar, apertar, perguntar onde é que dói? Aí, não faz nada, mal a gente senta."), conforme expressa um dos participantes desta pesquisa.

Influenciando a relação médico-paciente, devem ser levados em conta aspectos relacionados às condições de trabalho dos profissionais, como a precarização dos diversos aspectos necessários à execução do trabalho, de estruturação física do ambiente até a formação inadequada e incompleta do médico que se insere dentro de uma unidade básica de saúde e, também, o não cumprimento da carga horária, problemas que podem ser considerados recorrentes. ${ }^{15} \mathrm{Na}$ presente avaliação, a pontualidade e o cumprimento de horário não foram questionados diretamente nem para os próprios médicos nem para os pacientes entrevistados, mas foram apontados por um número elevado de pacientes. Quando perguntados sobre o que sugeririam para mudar a qualidade do serviço de saúde, as propostas mais apontadas pelos usuários referiram-se a aspectos relacionados à qualidade da assistência médica (melhorar a qualidade do médico, aumentar o número de profissionais, facilitar o sistema de encaminhamentos para especialidades e exames e até maior vigilância no desempenho profissional por parte dos gestores) e para aspectos relativos à estrutura física e serviços (melhor atendimento pelos funcionários e facilitação do sistema de agendamento). Nos dizeres de dois pacientes: [aqui a gente demora demais pra ser atendido, mal tem cadeira pra gente sentar enquanto espera.] [acho que tinha que ter mais médico aqui, ou então eles deviam cumprir o horário, ninguém controla]. A mesma pergunta dirigida aos médicos trouxe respostas parecidas. Apenas um médico respondeu que deveria haver uma melhora na formação médica para atuação em serviço público, somente dois sugeriram aumentar a quantidade de profissionais, número pequeno considerando-se a queixa de que as UBS geram grande demanda de usuários para cada médico, três fizeram referência à dificuldade de fornecer medicamentos ("aqui quase nunca tem remédios pra gente dar, e a gente sabe que muitas vezes o paciente nem vai comprar"), mas a proposta mais apresentada pelos médicos foi a de facilitar o encaminhamento para avaliação com especialistas ou para exames e a contrarreferência ("é muito difícil a gente conseguir encaminhar para um serviço mais especializado, na maioria das vezes a gente não consegue, ou se consegue, quase nunca temos a contrarreferência"). 
Uma das prerrogativas do SUS é a integralidade. Esta permite a articulação entre a atenção primária à saúde e o acesso aos meios de diagnóstico e atendimento em serviços de especialidade. Neste estudo, foi constatado que componentes dos dois grupos de entrevistados (médicos e usuários) citaram espontaneamente que suas necessidades não são atendidas: ou não conseguem os encaminhamentos necessários ou, quando conseguem, o processo é tão lento que não satisfazem mais às suas necessidades. Quanto à formação profissional, grande parte dos médicos entrevistados respondeu ter escolhido trabalhar naquela UBS por gostar de serviço público, pela remuneração, pela qualidade de vida e para adquirir experiência. De certo modo, assumem que não houve uma formação acadêmica para prepará-los para o ofício que já exercem, inclusive reconhecendo as limitações profissionais. Nos dizeres de um médico entrevistado, "tem um campo de aprendizado muito grande, dá pra ver muita coisa (...) que eu dizia: 'ah! Nunca vou ver isso' e eu vejo bastante aqui".

Na formalização da relação médico-paciente em seus diferentes âmbitos, as duas partes componentes têm expectativas diferentes e, às vezes, até mesmo conflitantes: o médico valoriza a evolução clínica e o tratamento instituído, dentro de modelos historicamente consagrados, ao passo que o paciente tem expectativas mais gerais, ligadas a aspectos mais subjetivos. ${ }^{1,23}$

Quando analisadas as visões dos médicos e dos usuários, ambos inseridos num mesmo serviço de saúde, são observadas diferenças de referencial, cada qual interpretando de maneira distinta o processo saúde-doença e também os serviços de saúde. As percepções dos usuários, em geral, são mais desfavoráveis em comparação às dos profissionais ${ }^{24}$ (paciente: A colega minha falou que ela entrou lá no consultório e ele [o médico] ficou conversando com os outros, isso é normal? Pra mim não é normal não, você atende a pessoa e enche de gente lá dentro do consultório?"/médico: "apesar de algumas dificuldades, aqui dá pra fazer um bom atendimento, é, em geral, os pacientes aqui são bem atendidos"). Essa percepção mais desfavorável dos pacientes pode ser constatada mesmo no caso de médicos que vivem a experiência de serem pacientes, ou seja, quando se trocam os papéis, trocam-se as expectativas. ${ }^{25}$ Possivelmente, isto se explica pelo fato do médico não dar atenção às múltiplas dimensões humanas que regem a relação médico-paciente. É neste contexto que os médicos entrevistados se inserem, pois, apesar de mencionarem uma lista grande de problemas que dificultam seu trabalho, dentre os quais a grande demanda de pacientes nos serviços, a dificuldade de encaminhamento, as falhas estruturais físicas e humanas nas UBS, e até mesmo a falta de resolubilidade, apenas dois médicos afirmaram não ser possível atender bem naquele local. Todos os demais não reconheceram que tantos obstáculos comprometem o bom atendimento.

Uma boa relação médico-paciente tem efeitos positivos na qualidade dos serviços de saúde, na aderência ao tratamento, no estado de saúde do paciente e na satisfação do usuário e do profissional. ${ }^{26,27}$ A empatia profissional pode influenciar até mesmo na resposta imune do paciente e encurtar o tempo de evolução da doença ou atenuar a sua morbidade. ${ }^{27}$ Evidentemente que, para tais resultados, o profissional médico tem que estar preparado para abordar o paciente em sua integralidade.

Pode-se dizer que a relação médico-paciente está ligada à formação profissional e também com o modelo de saúde instituído. É preciso que o médico perceba o paciente em sua integralidade e isto requer que o currículo educacional contenha os elementos básicos dos aspectos éticos e de comunicação, como já é realidade em algumas escolas médicas do mundo. ${ }^{25}$ 
Algumas limitações deste estudo devem ser consideradas. Embora sejam discutidos aspectos ligados à relação médico-paciente, cujo tema tem um corpo teórico mais específico, as entrevistas não focaram tantos atributos direcionados para uma análise mais precisa da relação entre as partes. Outra limitação é o fato de não ter sido avaliado o tempo de permanência dos profissionais em cada UBS estudada, o que pode refletir, indiretamente, na relação já historicamente estabelecida entre cada profissional de saúde e a demanda de pacientes daquela unidade. Sabe-se, porém, que todos os profissionais estavam locados em suas unidades há não muito tempo.

Apesar de algumas limitações, este trabalho permite reflexões sobre as relações estabelecidas entre o paciente, o profissional de saúde e a própria visão destes sobre as Unidades Básicas de Saúde, e estimula discussão sobre as expectativas essencialmente subjetivas do paciente, o papel do médico quanto a satisfazer tais expectativas, a formação profissional, dentre outros. O estudo espera, portanto, provocar discussão e gerar novos estudos que contribuam para uma transformação nas diferentes dimensões da prática médica.

\section{Considerações finais}

Uma boa relação entre os usuários e os serviços de saúde é fundamental para a satisfação dos pacientes e isto impõe, dentre tantos atributos, um atendimento médico centrado no indivíduo, de forma integral e em respeito à subjetividade de suas expectativas, um comprometimento dos profissionais, uma formação profissional adequada e o desenvolvimento de habilidades e competências para lidar melhor com o tempo de consulta e com a ética, e maiores cuidados com aspectos infraestruturais dos serviços.

Este estudo não tem por pretensão generalizar as afirmações dos respondentes ou possíveis conclusões que delas forem abstraídas. Os resultados aqui apresentados têm um caráter mais indicativo das tendências e peculiaridades que se estabelecem na relação médico-paciente nas unidades básicas de saúde estudadas.

No âmbito de uma consulta, é importante que se coloquem em prática, com habilidade e competência, alguns atributos que tornam a consulta mais eficaz para assegurar um melhor cuidado à pessoa. Para facilitar a relação médico-paciente, faz-se necessário um processo educativo que valorize um modelo de comunicação bidirecional, que permite uma relação de empatia e com a participação efetiva do paciente.

Este estudo traz uma reflexão para uma medicina de caráter complexo, que aborde conceitos éticos, antropológicos, metafísicos e epistemológicos. Aponta a necessidade de uma formação profissional que contemple o ser humano como sujeito de escolhas, o qual está inserido num contexto sociocultural que Ihe é singular.

\section{Referências}

1. Ballester D, Zucolotto SMC, Gannam SSA, Escobar, AMU. A inclusão da perspectiva do paciente na consulta médica: um desafio na formação do médico. Rev Bras Educ Med. 2010;34(4):598-606. DOI: http://dx.doi.org/10.1590/S010055022010000400016

2. Lopes JMC, Curra LCD. A importância do afeto na conduta do Médico de Família e Comunidade. Rev Bras Med Fam Comunidade. 2013;8(26):6-10. DOI: http://dx.doi.org/10.5712/rbmfc8(26)393 
3. Minayo MCS. Interdisciplinariedad y pensamiento complejo em el área de la salud. Salud Colectiva. 2008;4(1):5-8.

4. Lown B. The Lost Art of Healing: Practicing Comparision in Medicine. Boston: Houghton Mifflin Company; 1996.

5. Balint M. O Médico, seu paciente e a doença. São Paulo: Atheneu; 2007. p.291.

6. Carrió FB, Dohms M. Relação clínica na prática do médico de família. In: Gusso G, Lopes JMC. Tratado de Medicina de Família e Comunidade. São Paulo: Artmed; 2012, p.124-33.

7. Chinato IB, D’Agostini CL, Marques RR. A relação médico-paciente e a formação de novos médicos: análises de vivências de hospitalização. Rev Bras Med Fam Comunidade 2012;7(22):27-34. DOI: http://dx.doi.org/10.5712/rbmfc7(22)289

8. Lopes JMC. Consulta e abordagem centrada na pessoa. In: Gusso G, Lopes JMC. Tratado de Medicina de Família e Comunidade: princípios, formação e prática. Porto Alegre: Artmed; 2012, p.113-23.

9. Brunello MEF, Ponce MAZ, Assis EG, Andrade RLP, Scatena LM, Palha PF, et al. O vínculo na atenção à saúde: revisão sistematizada na literatura, Brasil (1998-2007). Acta Paul Enferm. 2010;23(1):131-5. DOI: http://dx.doi.org/10.1590/S010321002010000100021

10. Bosi MLM, Uchimura KY. Avaliação qualitativa de programas de saúde: contribuições para propostas metodológicas centradas na integridade e na humanização. In: Bosi MLM, Mercado-Martinez FJ, org. Avaliação Qualitativa de Programas de Saúde. Enfoques Emergentes. Petrópolis: Editora Vozes; 2006, p.87-117.

11. Fontanella BJB, Melo, DG, Germano CMR, Watanabe BM, et al. Iniciação científica com pesquisas qualitativas: relato de experiência de um grupo de professores e alunos de Medicina. Rev Bras Med Fam Comunidade. 2011;6(21):275-82. http://dx.doi.org/10.5712//rbmfc6(21)411

12. Adami NP, Maranhão AMSA. Qualidade dos serviços de saúde: conceitos e métodos avaliativos. Acta Paul Enferm. 1995;8(4):47-55.

13. Williams B. Patient satisfaction: a valid concept? Soc Sci Med. 1994;38(4):509-16. DOI: http://dx.doi.org/10.1016/0277-9536(94)90247-X

14. Vitolo MR, Gama CM, Campagnolo PD. Frequency of public child care service use and associated factors. J. Pediatr (Rio J). 2010;86(1):80-4.

15. Ribeiro JM, Siqueira SAV, Pinto, LFS. Avaliação da atenção à saúde da criança (0-5 anos) no PSF de Teresópolis (RJ) segundo a percepção dos usuários. Ciênc Saúde Coletiva. 2010;15(2):517-27 DOI: http://dx.doi.org/10.1590/S141381232010000200028

16. Cruz Neto O. Dificuldades da relação médico-paciente diante das pressões do "mercado de saúde". Ciênc Saúde Coletiva. 2003;8(1):307-8. DOI: http://dx.doi.org/10.1590/S1413-81232003000100023

17. Bodenheimer T. The future of primary care: transforming practice. N Engl J Med. 2008;359(20):2086-9. DOI: http://dx.doi. org/10.1056/NEJMp0805631

18. Marvel MK, Epstein RM, Flowers K, Beckman HB. Soliciting the patient's agenda: have we improved? JAMA. 1999;281(3):283-7. PMID: 9918487. DOI: http://dx.doi.org/10.1001/jama.281.3.283

19. Blau JN. Time to let the patient speak. BMJ. 1989;298(6665):39. PMID: 2492851. DOI: http://dx.doi.org/10.1136/ bmj.298.6665.39

20. Langewitz W, Denz M, Keller A, Kiss A, Rüttimann S, Wössmer B. Spontaneous talking time at start of consultation in outpatient clinic: cohort study. BMJ. 2002;325(7366):682-3. PMID:2492851. DOI: http://dx.doi.org/10.1136/bmj.325.7366.682

21. Albuquerque ABB, Bosi MLM. Visita domiciliar no âmbito da Estratégia Saúde da Família: percepções de usuários no Município de Fortaleza, Ceará, Brasil. Cad Saúde Pública. 2009;25(5):1103-12. DOI: http://dx.doi.org/10.1590/S0102$-311 \times 2009000500017$

22. Arroyo CS. Qualidade de serviços de assistência à saúde: o tempo de atendimento da consulta médica. [Tese de doutorado]. Ribeirão Preto, Faculdade de Economia, Administração e Contabilidade, Universidade de São Paulo; 2007.

23. Caprara A, Rodrigues J. A relação assimétrica médico-paciente: repensando o vínculo terapêutico. Ciênc Saúde Coletiva. 2004;9(1):139-46. DOI: http://dx.doi.org/10.1590/S1413-81232004000100014 
24. Van Stralen CJ, Belisário SA, Van Stralen TBS, Lima AMD, Massote AW, Oliveira CDL. Percepção dos usuários e profissionais de saúde sobre atenção básica: comparação entre unidades com e em saúde da família na Região Centro-Oeste do Brasil. Cad Saúde Pública. 2008;24(sup1):S148-58.

25. Caprara A, Franco ALS. A relação paciente-médico: para uma humanização da prática médica. Cad Saúde Pública. 1999;15(3):647-54. DOI: http://dx. doi.org/10.1590/S0102-311X1999000300023

26. Francis V, Korsch BM, Morris MJ. Fallos em la comunicacion medico-paciente. Respuesta de los pacientes a las recomendaciones del medico. In: White KL, Frenk J, Ordóñez C, Paganini JM, Starfield B. Investigaciones sobre servicios de salud: una antología. Washington: Organización Panamericana de La Salud; 1992.

27. Rakel DP, Hoeft TJ, Barrett BP, Chewning BA, Craig BM, Niu M. Practitioner empathy and the duration of the common cold. Fam Med. 2009;41(7):494-501. 